\title{
Early results on the use of biomaterials as adjuvant to abdominal wall closure following cytoreduction and hyperthermic intraperitoneal chemotherapy
}

\author{
Cherif Boutros, Ponandai Somasundar, N Joseph Espat ${ }^{*}$
}

\begin{abstract}
Background: Hyperthermic chemotherapy applies thermal energy to both abdominal wall as well as the intraabdominal viscera. The combination of the hyperthemia, chemotherapy and cytoreductive surgery (CRS) is associated with a defined risk of abdominal wall and intestinal morbidity reported to be as high as 15\%, respectively to date, no studies have evaluated the use of biomaterial mesh as adjuvant to abdominal wall closure in this group of patients. In the present report, we hypothesized that post HIPEC closure with a biomaterial can reduce abdominal wall morbidity after CRS and hyperthermic intraperitoneal chemotherapy.
\end{abstract}

Materials and methods: All patients treated with HIPEC in a tertiary care center over 12 months (2008-2009) period were included. Eight patients received cytoreductive surgery followed by HIPEC for 90 minutes using Mitomycin C (15 mg q 45 minutes $\times 2$ ). Abdominal wall closure was performed using Surgisis (Cook Biotech.) mesh in an underlay position with $3 \mathrm{~cm}$ fascial overlap-closure. Operative time, hospital length of stay (LOS) as well as postoperative outcome with special attention to abdominal wall and bowel morbidity were assessed.

Results: Eight patients, mean age 59.7 ys (36-80) were treated according to the above protocol. The primary pathology was appendiceal mucinous adenocarcinoma $(n=3)$ colorectal cancer $(n=3)$, and ovarian cancer $(n=$ 2). Four patients (50\%) presented initially with abdominal wall morbidity including incisional ventral hernia $(n=3)$ and excessive abdominal wall metastatic implants $(n=1)$. The mean peritoneal cancer index $(P C I)$ was 8.75 . Twenty eight CRS were performed (3.5 CRS/patient). The mean operating time was 6 hours. Seven patients had no abdominal wall or bowel morbidity, the mean LOS for these patients was 8 days. During the follow up period (mean 6.3 months), one patient required exploratory laparotomy 2 weeks after surgery and subsequently developed an incisional hernia and enterocutaneous fistula.

Conclusion: The use of biomaterial mesh in concert with HIPEC enables the repair of concomitant abdominal wall hernia and facilitates abdominal wall closure following the liberal resection of abdominal wall tumors. Biomaterial mesh prevents evisceration on repeat laparotomy and resists infection in immunocompromised patients even when associated with bowel resection.

\section{Introduction}

Hyperthermic intraperitoneal chemotherapy (HIPEC) has emerged as an effective method of managing peritoneal carcinomatosis for different abdominal malignancies particularly of colorectal and ovarian origin [1-3].

\footnotetext{
* Correspondence: jespat@hepaticsurgery.com

Hepatobiliary and Surgical Oncology, Roger Williams Medical Center, Providence, RI, USA
}

Most HIPEC treated patients have had prior abdominal surgeries and a substantial group of them present with abdominal wall morbidities including incisional hernia prior to HIPEC therapy.

Multiple studies have reported that chemotherapy administration impairs wound healing and that there is associated increase in wound complications in chemotherapy exposed patients $[4,5]$. The effects of HIPEC 


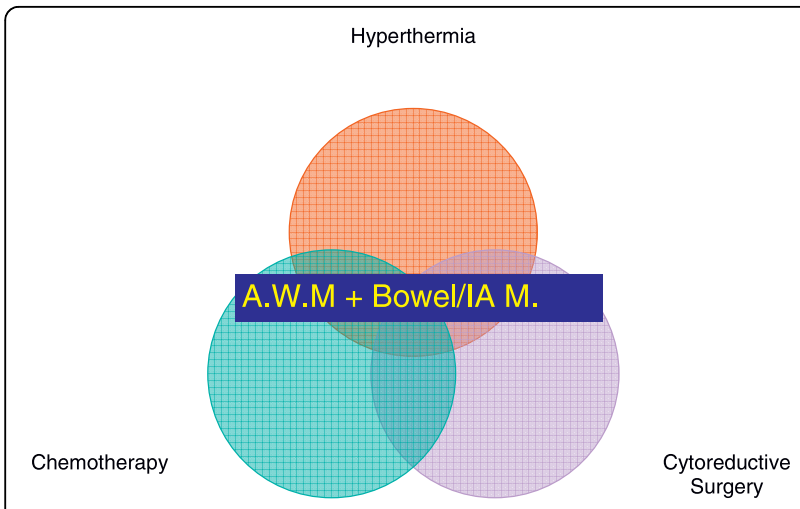

Figure 1 The association of hyperthermia, cytoreductive surgery and chemotherapy carry a considerable risk of abdmonial wall and bowel morbidity.

on wound healing are multiple (Figure 1): first HIPEC requires the concomitant use of chemotherapy and hyperthermia which are both known to increase cellular death and induce apoptosis [6,7]; second, tumor cytoreduction may require surgical resection of involved abdominal wall surfaces potentially compromising abdominal wall closure and strength.

HIPEC impairment of wound healing is not limited to the abdominal wall, but also anastomosed segments of the gastrointestinal tract after cytoreductive surgery bowel resections. In small animal models, HIPEC was found to significantly decrease both colonic anastomotic bursting pressure and abdominal wall strength in association with decreased local protein production $[8,9]$. In clinical studies, HIPEC was associated with a non negligible percentage (up to 15\%) of abdominal wall morbidity including; wound infection, dehiscence, evisceration and bowel morbidity including; anastomotic leak and intra-abdominal abscess (Table 1) [10,11].

Moreover, postoperative bowel morbidity often required reoperation [10], in this case abdominal wound healing already inhibited by HIPEC will be impaired by the infected milieu.

Biomaterial mesh has emerged as an attractive option for complex abdominal wall reconstruction providing an additional reinforcement to the abdominal wall with an

Table 1 Selected studies reporting abdominal wall morbidity (AWM) and bowel/intra-abdominal morbidity (Bowel/IA M)

\begin{tabular}{cccc}
\hline Study (year) & N & AWM & Bowel/IA M \\
\hline Franko (2008) [10] & 65 & $10.7 \%$ & $15.4 \%$ \\
Kianmanesh (2007) [2] & 43 & $11.6 \%$ & $13.9 \%$ \\
Stewart (2006) [11] & 110 & $15.4 \%$ & $6.3 \%$ \\
Sugarbaker (2006) [29] & 356 & $3 \%$ & $5.47 \%$ \\
Witkamp (2001) [30] & 29 & $3 \%$ & $3 \%$ \\
\hline
\end{tabular}

absorbable material resistant to infection and with potential remodeling to host own tissue. Therefore, we used a biomaterial mesh to reinforce the abdominal wall closure at the end of CRS-HIPEC procedure. We hypothesized that this approach could minimize the rate of postoperative abdominal wall complications reported after CRS-HIPEC.

\section{Methods}

Under institutional IRB approval, all patients treated with HIPEC at a tertiary care center over a12 months interval (2008-2009) period were identified using a prospectively maintained departmental database. Records were reviewed for preoperative, operative and postoperative data. Pertinent information for analysis included gender, age, primary malignancy, number of previous abdominal operations, peritoneal cancer index [12], operative time and cytoreductive procedures performed. Follow-up data were obtained by review of clinic notes. Abdominal wall and bowel/intra-abdominal morbidities were ascertained during the immediate and postoperative period and subsequent clinic visits.

Data was recorded in a Microsoft Excel ${ }^{\circ}$ (Microsoft, Redmond, WA) database. Descriptive statistics, including means, standard deviations or counts and percentages were calculated.

\section{Operative technique}

Abdominal exploration was performed through midline laparotomy incision. Lysis of adhesions, when needed was performed, followed by assessment of the intraabdominal extent of the disease and the peritoneal cancer index (PCI). Appropriate cytoreductive surgery was then performed with an attempt for complete removal of all macroscopic tumor deposits on parietal and visceral peritoneal surfaces and resection of involved viscera.

After completion of the CRS, HIPEC was immediately performed during the same surgical procedure for all patients using a closed-abdomen technique (Figure 2). Two inflow catheters were placed under direct vision to the right and left upper quadrants above the liver and the spleen respectively and two outflow catheters were placed under direct vision on the right and left paracolic gutters. The inflow and outflow tubes were secured to the skin and connected to a cardiopulmonary bypass pump (Figure 3). A temperature needle probe was placed into one of the outflow catheters and the skin was closed using a running locked heavy nylon stitch, while the fascia was left open.

After priming the circuit, a flow of 2.0-2.4 L/minute of DIANEAL PD-2, dextrose $1.5 \%{ }^{\circ}$ (Baxter, Deerfield, IL) solution containing Mitomycin $\mathrm{C}$ was obtained and maintained during the whole HIPEC time. Mitomycin $\mathrm{C}$ was used at a standard dose of $15 \mathrm{mg} \mathrm{q} 45$ minutes $\times 2$ 


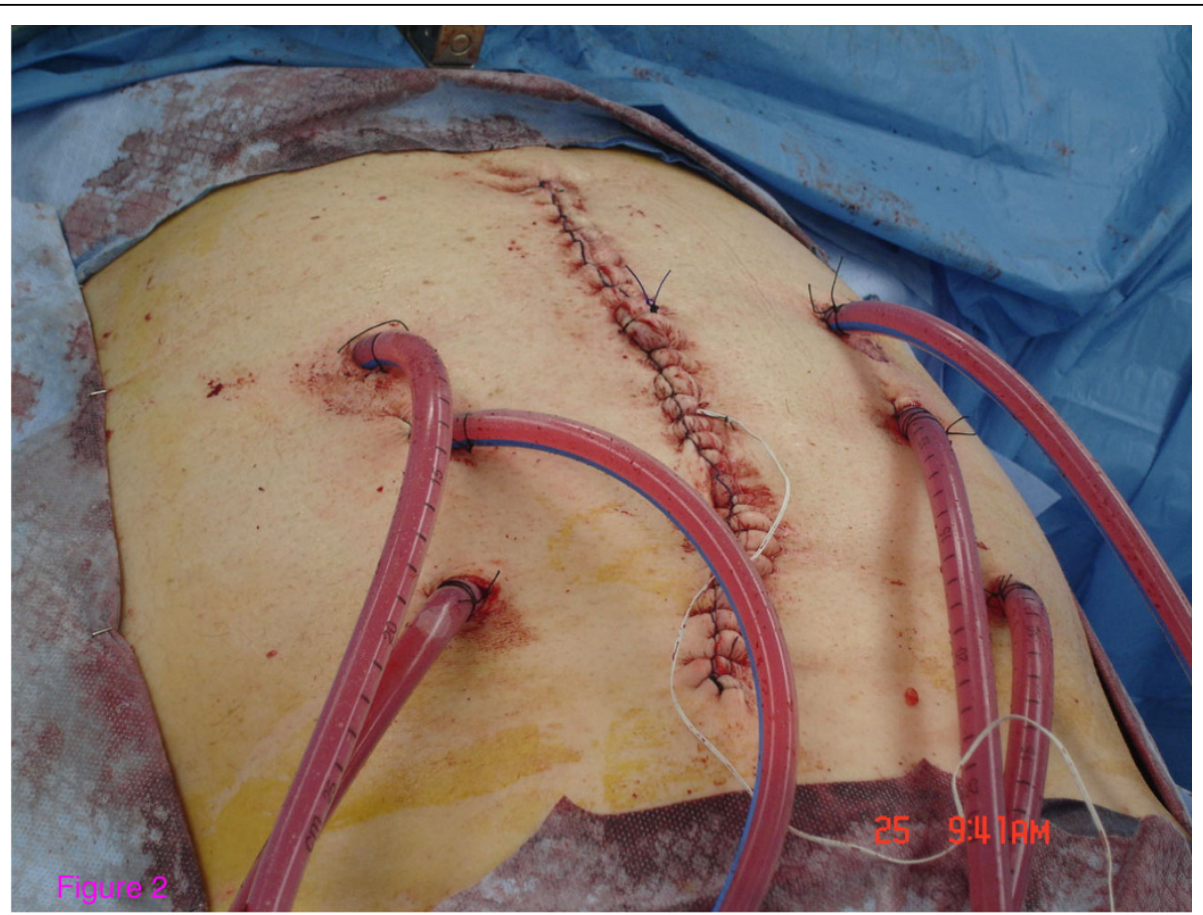

Figure 2 Using a closed technique: Two inflow and four outflow catheters were used for HIPEC administration. Only the skin of the surgical wound is temporarily closed.

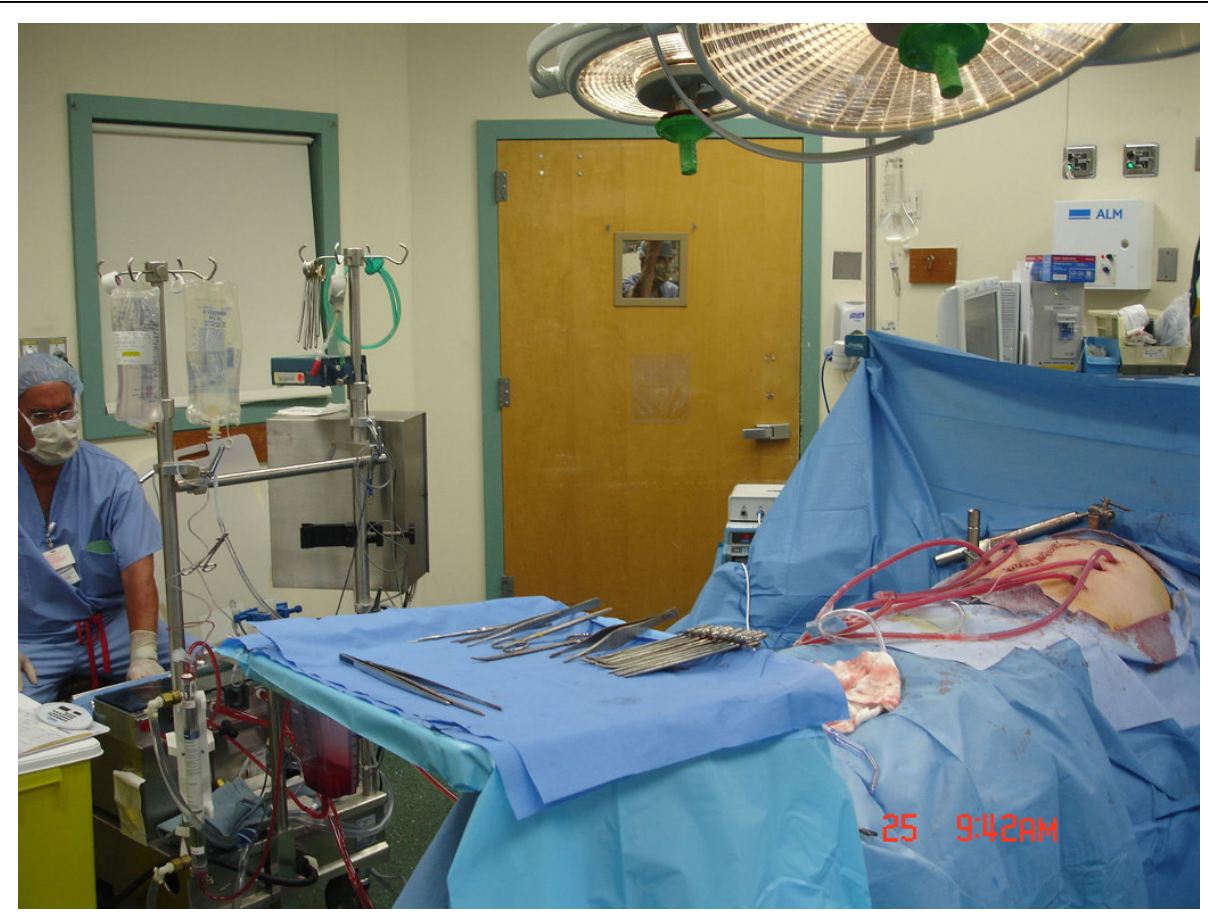

Figure 3 Using a cardiopulmonary bypass machine, The HIPEC circuit is completed. 
for all patients. Hyperthermia was obtained by a heating unit in the cardiopulmonary bypass pump maintaining inflow temperature at $42^{\circ} \mathrm{C}$, while the abdominal temperature was continuously checked by the needle probe to confirm a minimu of $41^{\circ} \mathrm{C}$ in the outflow. A total HIPEC time of 90 minutes was applied to all patients.

At the end of the 90 minutes dwell time, the HIPEC solution was retrieved through the cardiopulmonary pump outflow catheters and disposed; the abdominal cavity was flushed with the effluent removed via the outflow catheters and the abdominal incision was reopened.

The abdominal wall was closed using a $20 \times 20 \mathrm{~cm}$ piece of Surgisis ${ }^{\oplus}$ mesh (Cook Biotech, West Lafayette, IN). Abdominal wall closure was performed by the same surgeon performing the CRS-HIPEC procedure. Surgisis mesh was placed in underlay position and secured to the anterior abdominal wall using circumferential transfascial absorbable sutures (\#1 PDS) $2 \mathrm{~cm}$ apart. When possible, the native fascia was closed over the Surgisis mesh using absorbable sutures and the skin was closed using skin stapler.

\section{Results}

The RWMC HIPEC program started on June 2008; over one year period eleven patients received HIPEC, including three patients who received totally laparoscopic HIPEC for persistent PET scan evident activity from colorectal cancer in the mesenteric or retroperitoneal lymph nodes after standard adjuvant chemotherapy. Eight patients received exploratory laparotomy, CRS and HIPEC and were included in this study (Table 2).

Patients' mean age was 59.7 years (36-80), M: F ratio was $1: 1$ and the origin of the primary malignant disease was colorectal $(\mathrm{n}=4)$, appendiceal $(\mathrm{n}=2)$ and ovarian $(\mathrm{n}=2)$. All patients had prior abdominal surgeries and three patients had > two prior abdominal explorations. Peritoneal cancer index varied from $0-18$ with a mean of 8.75 . Prior to HIPEC therapy, four patients $(4 / 8,50 \%)$ presented with abdominal wall morbidities including incisional hernias $(\mathrm{n}=3)$ and abdominal wall metastatic implants $(\mathrm{n}=1)$.

A total 28 CRS procedures were performed (average 3.5 /patient). One third of the CRS included bowel resection-anastomosis $(9 / 28,32 \%)$ all performed by linear staplers.

Mean total surgical procedure time was $5 \mathrm{~h} 49 \mathrm{~min}$ utes $\pm 1 \mathrm{~h} 10$ minutes including 90 minutes devoted for HIPEC (Table 3). There was no peri-operative mortality and no postoperative neutropenia. Seven patients had neither abdominal wall nor bowel/intra-abdominal morbidities with a mean length of stay of 8 days (range 416). All patients were followed in subsequent clinic visits, the mean follow up was 6.3 months.
Table 2 Patient characteristics

\begin{tabular}{|c|c|c|c|c|c|}
\hline Age & Sex & ASA & Pathology & Prior surgery & $\mathrm{PCl}$ \\
\hline 53 & M & 2 & $\begin{array}{c}\text { MAC } \\
\text { Appendix }\end{array}$ & Appendectomy & 18 \\
\hline 70 & M & 2 & $\begin{array}{l}\text { Colorectal } \\
\text { cancer }\end{array}$ & $\begin{array}{c}\text { Open colectomy and ventral } \\
\text { hernia repair }\end{array}$ & 3 \\
\hline 59 & F & 3 & $\begin{array}{l}\text { Ovarian } \\
\text { Cancer }\end{array}$ & Three midline Ex Lap. & 12 \\
\hline 57 & F & 2 & $\begin{array}{c}\text { MAC } \\
\text { Appendix }\end{array}$ & Appendectomy & 0 \\
\hline 72 & M & 3 & $\begin{array}{l}\text { Colorectal } \\
\text { Cancer }\end{array}$ & Open colectomy & 9 \\
\hline 80 & F & 3 & $\begin{array}{l}\text { Ovarian } \\
\text { Cancer }\end{array}$ & Hysterectomy & 10 \\
\hline 36 & $\mathrm{~F}$ & 2 & $\begin{array}{c}\text { MAC } \\
\text { Appendix }\end{array}$ & TAHBSO & 15 \\
\hline 51 & M & 3 & $\begin{array}{l}\text { Colorectal } \\
\text { cancer }\end{array}$ & Open colectomy + 4 Ex. Lap. & 3 \\
\hline
\end{tabular}

PCI: Peritoneal cancer index; MAC: Mucinous adenocarcinoma; TAHBSO: total abdominal hysterectomy and bilateral salpingo-oopherectomy; Ex. Lap.: Exploratory laparotomy.

One patient required re-exploration two weeks after HIPEC procedure and subsequently developed incisional hernia and enterocutaneous fistula. This patient is a 59 year old patient with ovarian cancer and three prior abdominal surgeries including two debulking procedures. Prior to HIPEC procedure, the patient physical examination was significant for extensive abdominal wall metastatic implants.

The patient PCI was 12 and CRS included bowel resection/anastomosis $\mathrm{x} 2$, splenectomy and abdominal wall resection. The patient post-operative period was marked by respiratory failure requiring reintubation. On post-operative day 15 , a brown discharge was noted from the surgical incision and a decision for surgical reexploration was made.

Upon re-exploration, the previously placed Surgisis mesh was intact and easily dissected from the underlying bowel, prior anastomoses were intact and there was no evidence of gastrointestinal leak. Blood culture was obtained when the postoperative wound discharge was initially noted and intravenous antibiotic therapy was

Table 3 Outcome of patients after HIPEC surgery

\begin{tabular}{cccc}
\hline Surgical time $(\mathbf{m n})$ & AWM & Bowel/IA M & LOS (D) \\
\hline 410 & $\varnothing$ & $\varnothing$ & 9 \\
310 & $\varnothing$ & $\varnothing$ & 4 \\
380 & Dehiscence & ECF & 120 \\
280 & $\varnothing$ & $\varnothing$ & 6 \\
370 & $\varnothing$ & $\varnothing$ & 5 \\
430 & $\varnothing$ & $\varnothing$ & 16 \\
230 & $\varnothing$ & $\varnothing$ & 10 \\
380 & $\varnothing$ & $\varnothing$ & 6 \\
\hline
\end{tabular}

AWM: Abdominal wall morbidity; Bowel/IA M: Bowel and intra-abdominal morbidity; LOS: Length of stay; ECF: Enterocutaneous fistula 
started imperatively with the hypothesis of sepsis. During re-exploration cultures from the peritoneal fluid were also obtained; both blood and peritoneal cultures did not support an infectious etiology. A dramatic lysis of residual tumor implants was noted producing a melted brown discharge. A new Surgisis mesh was placed and the fascia was closed as previously described. Subsequently, the patient developed wound dehiscence with no evisceration as well as enterocutaneous fistula. A negative pressure dressing was applied to the abdominal wound and the patient was discharged home three months after the HIPEC procedure.

\section{Discussion}

The introduction of biomaterial mesh had revolutionized the field of abdominal wall closure [13,14]. Broadly grouped, biomaterial mesh is either human allograft or xenograft and dermal or non dermal in origin. Specific guidelines for specific biomaterial mesh selection for a given case remain to be defined; however in general it is accepted that for complex and contaminated cases, biomaterial mesh offers a viable substitute to the patient's own tissue. In particular the use of biomaterial mesh has been described to be clinically meaningful when the host native abdominal fascia is insufficient for closure without tension, ie (loss of abdominal domain), when there is a lack of viable tissue and a components separation is not technically feasible, or the field is contaminated or potentially contaminated and permanent synthetic mesh is relatively contraindicated [15]. Biomaterial meshes are known to be resistant to infection[16] and overcome the limitations of synthetic mesh for use in contaminated or potentially contaminated wounds, provide a tissue remodeling matrix, for host tissues and fibroblasts [17]. In this series, the potential role of biomaterial mesh as adjuvant to abdominal wall closure in the setting of significantly potential impaired abdominal wall wound healing following HIPEC, with or without prior incisional hernia or after cytoreductive surgery of abdominal wall metastatic implants was investigated. In cases, there was a clinical indication for mesh reinforcement due to weakened, lacking or non viable abdominal wall fascia; the choice of biomaterial mesh was supported by the presence of potential contamination or frank contamination subsequent to a procedure entering the gastrointestinal tract.

Surgisis mesh was utilized in all open HIPEC procedures. This biomaterial mesh is composed of lyophilized porcine small intestinal submucosa, is known to attract cells to the wound area and signaling surrounding tissues to grow across the scaffold [18]. The choice of this particular biomaterial mesh was based on the senior authors previous published experience with Surgisis
$[14,19]$ as well as the reports of others observing that Sugisis remodels into vascularized host tissue [17], thus allowing resistance to infection. Additionally, Surgisis is predominantley composed of collagen rather than elastin compared to dermal-based biomaterials; thus it is expected to result in less abdominal diathesis or hernia recurrence overtime [20].

All Surgisis meshes were placed in underlay position with a minimum of a bilateral $3 \mathrm{~cm}$ fascial overlap-closure using absorbable number one PDS transfascial sutures placed circumferentially no more than two $\mathrm{cm}$ apart. Underlay repairs, such as Rivers-Stoppa retro-rectus repair, have been reported to result in improved recurrence rate and allow for re-approximation of the midline, thus potentially improving the mechanical function of the abdominal wall [21,22].

The HIPEC protocol employed was the well-described regimen of single agent (Mitomycin C) at a dose (15 mg q 45 minutes $\times 2$ ) with a cumulative dwell time of 90 minutes, for all patients regardless of the origin of the primary malignancy and the body surface area of the patient [23]. The present protocol does not include a measurement of serum Mitomycin $C$ levels thus we are unable to discuss its pharmakodynamics in this setting, these data have been previously described by others $[24,25]$. Postoperatively, none of the patients in this series developed neutropenia which has reported to occur in up to $39 \%$ of HIPEC patients using Mitomycin C at a higher dose[26]. It should be emphasized that though there are multiple series reporting the use of different chemotherapeutic agent (s) and different doses during HIPEC, there is no consensus statement or general agreement on a single universal protocol. Currently, efforts are undergoing to create a registry database for all active HIPEC programs in USA allowing outcome analysis to elucidate this still evolving topic.

Seven of eight patients included in this study did not develop abdominal wall or bowel/intra-abdominal morbidities postoperatively and were discharged home after a mean length of stay of eight days. A single patient did sustain the complication of suspicious for enterocutaneous fistula wound discharge with associated with respiratory failure 7 days after HIPEC necessitating reexploration. Upon re-exploration, the integrity of the abdominal wall and gastrointestinal were verified. The Surgisis mesh was found intact and the bowel was easily dissected from the mesh as has been previously described in experimental models $[27,28]$. At operation, the patient was found to have extensive tumor necrosis from unresectable pelvic mass; because of potential compromised rectal wall, a loop diverting ostomy was created. The abdominal wall fascia was closed again with a new Surgisis mesh to prevent evisceration. Not unexpectedly, the native fascia later dehisced, and the 
patient developed and enterocutaneous fistula, however, the new Surgisis mesh was allowed to granulate and subsequently remodeled, allowing non surgical management.

Subsequent to HIPEC, perioperative abdominal wall and bowel/intra-abdominal complications may require surgical exploration [10]. In this population re-exploration carries a high risk to both the abdominal wall recently exposed to hyperthermic chemotherapy and the bowel is usually firmly adherent to the abdominal with associated evisceration and/or the formation of enterocutaneous fistulae. The use of synthetic mesh as adjuvant to abdominal wall closure in such circumstances is contraindicated due to the potentially infected and contaminated environment.

The authors would emphasize the limitations of this study. This series represents early results of an abdominal wall reconstructive technique that in our experience was found to be safe and effective in preventing abdominal wall complications in a cohort of patients at high risk in the short term postoperative period. However, this report represents the experience of a single institution with a modest cohort and a short follow up. Long term follow up is needed before complete success for the use of biomaterial mesh can be declared to more define the role for biomaterial mesh in treatment and prevention of surgical site hernia following HIPEC and cytoreductive surgery. This may be limited by the inherent limited survival of many patients with disseminated peritoneal malignancy.

In this small series biomaterial mesh was applied at the end of all CRS-HIPEC procedure and showed a beneficial effect in minimizing early postoperative abdominal wall complications. However, extrapolating these results to be applied to all CRS-HIPEC patients as a universal approach will probably reveal that only a group of high risk patients including those with multiple abdominal wall surgeries, preoperative abdominal wall hernia or metastatic implants to the abdominal wall will benefit from such approach.

The selection of Surgisis mesh, was based on results of other studies reporting its resistant to infection and total remodeling in settings other than HIPEC. It will be necessary to document remodeling in this setting by histopathology incorporating tissue samples during the various stages of wound healing at distinct intervals after HIPEC with and without Surgisis mesh abdominal wall reconstruction.

Finally, the HIPEC protocol used only Mitomycin C as single agent chemotherapy. Although, chemotherapeutic agents share an inhibitory effect on wound healing, it is possible, although not yet investigated that in the setting of HIPEC, that this known inhibitory effect varies significantly between different agents.

\section{Conclusion}

In this series, the use of a biomaterial mesh for abdominal wall reconstruction following CRS and HIPEC enabled the repair of concomitant abdominal wall hernias and facilitated liberal resection of abdominal wall tumors. Biomaterial mesh prevented evisceration on repeat laparotomy and resists infection in immunocompromised patients even when associated with bowel resection.

\section{Authors' contributions}

$\mathrm{CB}$ assisted with acquisition of data and drafting the manuscript, PS assisted with revision of the manuscript, NJE assisted with study design and revision of the manuscript. All authors read and approved the final manuscript

\section{Competing interests}

The senior author, Dr. Espat has previously received honoraria from Cook as a speaker.

Received: 7 March 2010 Accepted: 20 August 2010

Published: 20 August 2010

\section{References}

1. Esquivel J, Sticca R, Sugarbaker $P$, Levine E, Yan TD, Alexander R, Baratti D, Bartlett D, Barone R, Barrios P, Bieligk S, Bretcha-Boix P, Chang CK, Chu F, Chu Q, Daniel S, de Bree E, Deraco M, Dominguez-Parra L, Elias D, Flynn R, Foster J, Garofalo A, Gilly FN, Glehen O, Gomez-Portilla A, Gonzalez-Bayon L, Gonzalez-Moreno S, Goodman M, Gushchin V, et al: Cytoreductive surgery and hyperthermic intraperitoneal chemotherapy in the management of peritoneal surface malignancies of colonic origin: a consensus statement. Society of Surgical Oncology. Ann Surg Oncol 2007, 14(1):128-33.

2. Kianmanesh R, Scaringi S, Sabate JM, Castel B, Pons-Kerjean N, Coffin B, Hay JM, Flamant Y, Msika S: Iterative cytoreductive surgery associated with hyperthermic intraperitoneal chemotherapy for treatment of peritoneal carcinomatosis of colorectal origin with or without liver metastases. Ann Surg 2007, 245(4):597-603.

3. Lim MC, Kang S, Choi J, Song YJ, Park S, Seo SS, Park SY: Hyperthermic intraperitoneal chemotherapy after extensive cytoreductive surgery in patients with primary advanced epithelial ovarian cancer: interim analysis of a phase II study. Ann Surg Oncol 2009, 16(4):993-1000.

4. Shukla VK, Pandey M, Das PC, Tiwary SK, Chansouria JP, Kumar M, Singh G, Pandey SK: Effect of 5-fluorouracil and mitomycin on the healing of intestinal anastomosis. Chemotherapy 2007, 53(4):275-81.

5. Gordon CR, Rojavin Y, Patel M, Zins JE, Grana G, Kann B, Simons R, Atabek $U$ : A review on bevacizumab and surgical wound healing: an important warning to all surgeons. Ann Plast Surg 2009, 62(6):707-9.

6. Adachi $S$, Kokura S, Okayama $T$, Ishikawa $T$, Takagi $T$, Handa O, Naito $Y$, Yoshikawa T: Effect of hyperthermia combined with gemcitabine on apoptotic cell death in cultured human pancreatic cancer cell lines. Int J Hyperthermia 2009, 25(3):210-9.

7. Wang X, Gao XH, Li X, Hong Y, Qi R, Chen HD, Zhang L, Wei H: Local hyperthermia induces apoptosis of keratinocytes in both normal skin and condyloma acuminata via different pathways. Apoptosis 2009.

8. Aarts F, Bleichrodt RP, de Man B, Lomme R, Boerman OC, Hendriks T: The effects of adjuvant experimental radioimmunotherapy and hyperthermic intraperitoneal chemotherapy on intestinal and abdominal healing after cytoreductive surgery for peritoneal carcinomatosis in the rat. Ann Surg Oncol 2008, 15(11):3299-307.

9. Pelz JO, Doerfer J, Decker M, Dimmler A, Hohenberger W, Meyer T: Hyperthermic intraperitoneal chemoperfusion (HIPEC) decrease wound strength of colonic anastomosis in a rat model. Int J Colorectal Dis 2007, 22(8):941-7.

10. Franko J, Gusani NJ, Holtzman MP, Ahrendt SA, Jones HL, Zeh HJ, Bartlett DL: Multivisceral resection does not affect morbidity and survival after cytoreductive surgery and chemoperfusion for carcinomatosis from colorectal cancer. Ann Surg Oncol 2008, 15(11):3065-72. 
11. Stewart JHt, Shen P, Russell GB, Bradley RF, Hundley JC, Loggie BL, Geisinger KR, Levine EA: Appendiceal neoplasms with peritoneal dissemination: outcomes after cytoreductive surgery and intraperitoneal hyperthermic chemotherapy. Ann Surg Oncol 2006, 13(5):624-34.

12. Sugarbaker PH, Jablonski KA: Prognostic features of 51 colorectal and 130 appendiceal cancer patients with peritoneal carcinomatosis treated by cytoreductive surgery and intraperitoneal chemotherapy. Ann Surg 1995, 221(2):124-32

13. Johnson EK, Paquette EL: Use of surgisis for abdominal wall reconstruction/closure in battlefield casualties during Operation Iraqi Freedom. Mil Med 2007, 172(10):1119-24.

14. Helton WS, Fisichella PM, Berger R, Horgan S, Espat NJ, Abcarian H: Shortterm outcomes with small intestinal submucosa for ventral abdominal hernia. Arch Surg 2005, 140(6):549-60, discussion 560-2.

15. Dasgupta R, Wales PW, Zuker RM, Fisher DM, Langer JC: The use of Surgisis for abdominal wall reconstruction in the separation of omphalopagus conjoined twins. Pediatr Surg Int 2007, 23(9):923-6.

16. Franklin ME Jr, Trevino JM, Portillo G, Vela I, Glass JL, Gonzalez JJ: The use of porcine small intestinal submucosa as a prosthetic material for laparoscopic hernia repair in infected and potentially contaminated fields: long-term follow-up. Surg Endosc 2008, 22(9):1941-6.

17. Hodde J, Hiles M: Constructive soft tissue remodelling with a biologic extracellular matrix graft: overview and review of the clinical literature. Acta Chir Belg 2007, 107(6):641-7.

18. De Ugarte DA, Puapong D, Roostaeian J, Gillis N, Fonkalsrud EW, Atkinson JB, Dunn JC: Surgisis patch tracheoplasty in a rodent model for tracheal stenosis. J Surg Res 2003, 112(1):65-9.

19. Ong ES, Helton WS, Jho D, Espat NJ: SURGISIS-assisted surgical site control in the delayed repair of a complex bile duct injury after laparoscopic cholecystectomy. J Gastrointest Surg 2006, 10(2):202-6.

20. Gupta A, Zahriya K, Mullens PL, Salmassi S, Keshishian A: Ventral herniorrhaphy: experience with two different biosynthetic mesh materials, Surgisis and Alloderm. Hernia 2006, 10(5):419-25.

21. Novitsky YW, Porter JR, Rucho ZC, Getz SB, Pratt BL, Kercher KW, Heniford BT: Open preperitoneal retrofascial mesh repair for multiply recurrent ventral incisional hernias. J Am Coll Surg 2006, 203(3):283-9.

22. Temudom T, Siadati M, Sarr MG: Repair of complex giant or recurrent ventral hernias by using tension-free intraparietal prosthetic mesh (Stoppa technique): lessons learned from our initial experience (fifty patients). Surgery 1996, 120(4):738-43, discussion 743-4.

23. Beaujard AC, Glehen O, Caillot JL, Francois Y, Bienvenu J, Panteix G, Garbit F, Grandclement E, Vignal J, Gilly FN: Intraperitoneal chemohyperthermia with mitomycin $\mathrm{C}$ for digestive tract cancer patients with peritoneal carcinomatosis. Cancer 2000, 88(11):2512-9.

24. Jacquet $P$, Averbach $A$, Stephens $A D$, Stuart OA, Chang D, Sugarbaker PH: Heated intraoperative intraperitoneal mitomycin $C$ and early postoperative intraperitoneal 5-fluorouracil: pharmacokinetic studies. Oncology 1998, 55(2):130-8.

25. de Lima Vazquez V, Stuart OA, Mohamed F, Sugarbaker PH: Extent of parietal peritonectomy does not change intraperitoneal chemotherapy pharmacokinetics. Cancer Chemother Pharmacol 2003, 52(2):108-12.

26. Lambert LA, Armstrong TS, Lee JJ, Liu S, Katz MH, Eng C, Wolff RA, Tortorice ML, Tansey P, Gonzalez-Moreno S, Lambert DH, Mansfield PF: Incidence, Risk Factors, and Impact of Severe Neutropenia After Hyperthermic Intraperitoneal Mitomycin C. Ann Surg Oncol 2009.

27. Costa RG, Lontra MB, Scalco P, Cavazzola LT, Gurski RR: Polylactic acid film versus acellular porcine small intestinal submucosa mesh in peritoneal adhesion formation in rats. Acta Cir Bras 2009, 24(2):128-35.

28. Ansaloni L, Catena F, Coccolini F, Fini M, Gazzotti F, Giardino R, Pinna AD: Peritoneal adhesions to prosthetic materials: an experimental comparative study of treated and untreated polypropylene meshes placed in the abdominal cavity. J Laparoendosc Adv Surg Tech A 2009, 19(3):369-74.

29. Sugarbaker PH, Alderman R, Edwards G, Marquardt CE, Gushchin V, Esquivel J, Chang D: Prospective morbidity and mortality assessment of cytoreductive surgery plus perioperative intraperitoneal chemotherapy to treat peritoneal dissemination of appendiceal mucinous malignancy. Ann Surg Oncol 2006, 13(5):635-44.

30. Witkamp AJ, de Bree E, Kaag MM, Boot H, Beijnen JH, van Slooten GW, van Coevorden F, Zoetmulder FA: Extensive cytoreductive surgery followed by intra-operative hyperthermic intraperitoneal chemotherapy with mitomycin- $C$ in patients with peritoneal carcinomatosis of colorectal origin. Eur J Cancer 2001, 37(8):979-84.

doi:10.1186/1477-7819-8-72

Cite this article as: Boutros et al:: Early results on the use of biomaterials as adjuvant to abdominal wall closure following cytoreduction and hyperthermic intraperitoneal chemotherapy. World Journal of Surgical Oncology 2010 8:72.

\section{Submit your next manuscript to BioMed Central and take full advantage of:}

- Convenient online submission

- Thorough peer review

- No space constraints or color figure charges

- Immediate publication on acceptance

- Inclusion in PubMed, CAS, Scopus and Google Scholar

- Research which is freely available for redistribution

Submit your manuscript at www.biomedcentral.com/submit
Biomed Central 